\title{
Plasma Processing to Mitigate Field Emission in LCLS-II 1.3 GHz SRF Cavities
}

\section{Plasma Cleaning to Reduce FE}

A collaboration between Fermilab, SLAC and ORNL is working to develop plasma processing for $1.3 \mathrm{GHz}$ TESLA shaped SRF cavities.

Hydrocarbon $\left(C_{x} H_{y}\right)$ contaminations and adsorbates lower the cavity surface work function $(\Phi)$ increasing Field Emission (FE). Plasma processing removes $C_{x} H_{y}$ allowing the cavity to operate at higher $\mathrm{E}_{\mathrm{acc}}$.

A $10 \%$ increase in $\Phi$ gives a $15 \%$ increase in $E_{\text {acc }}$ $\mathrm{O}_{2}+\mathrm{C}_{x} \mathrm{H}_{y} \rightarrow \mathrm{CO}+\mathrm{CO}_{2}+\mathrm{H}_{2} \mathrm{O}$

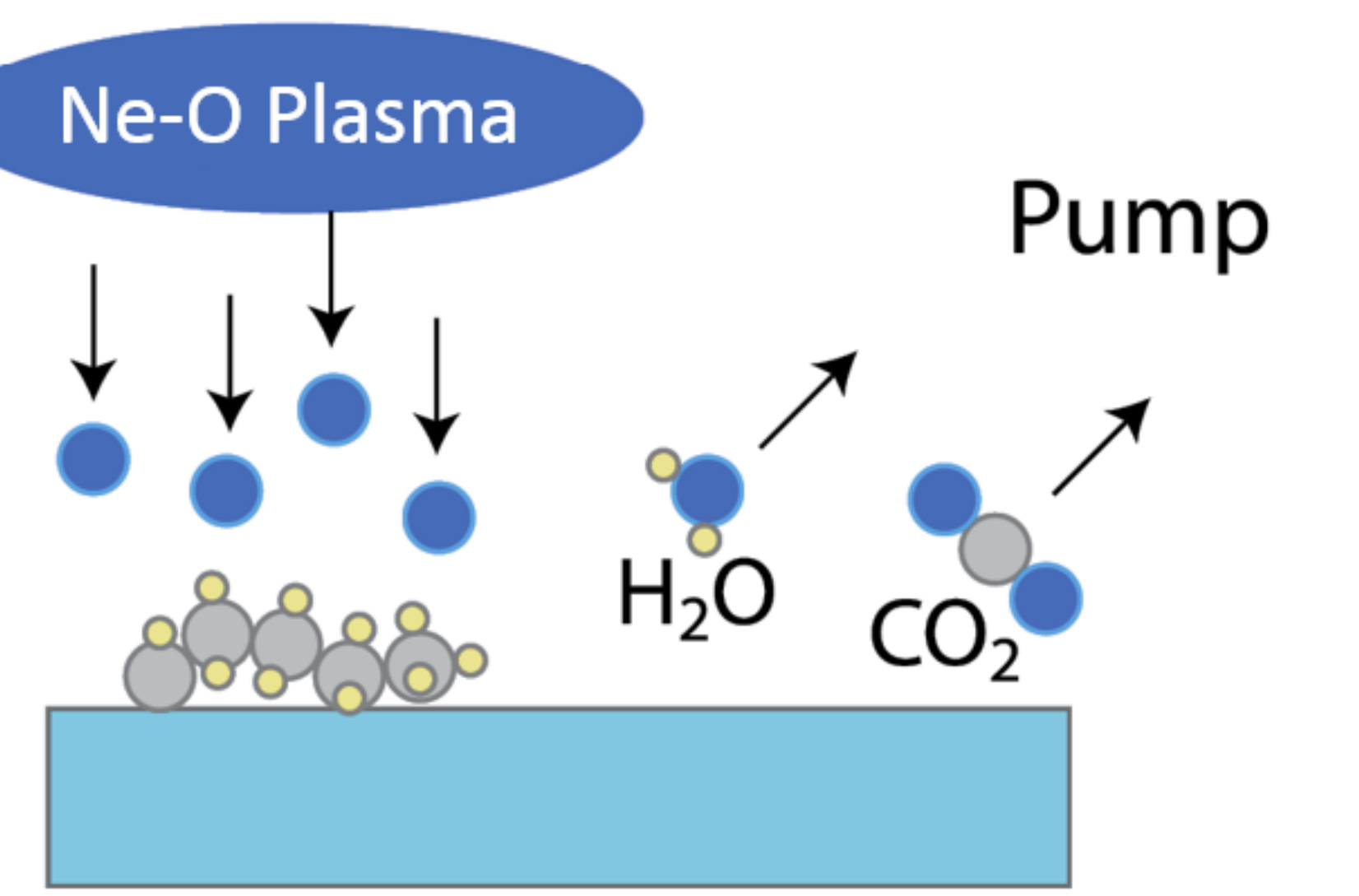

Plasma cleaning: glow discharge ignited at room temperature using an inert gas ( $\mathrm{Ne}$ or $\mathrm{Ar}$ ). A low \% of $\mathrm{O}_{2}$ is added to react with $C_{x} H_{y}$.

Cavity pressure: $70-200 m$ Torr.

M. Doleans et al. NIMA 812 (2016) 50-59

\section{Plasma ignition in LCLS-II cavities}

Glow discharge is ignited cell by cell, using cavity resonant modes.

New idea: ignite plasma using HOMs (Higher Order Modes): $1^{\text {st }}$ and $2^{\text {nd }}$ dipole pass-bands $\longrightarrow$ good coupling at room temperature, resulting in plasma ignition with only few watts. Dual tone excitation to transfer plasma:

plasma ignited in the central cell (mode 2D-1) $\longrightarrow$ transferred through adjacent cells using HOMs superposition.
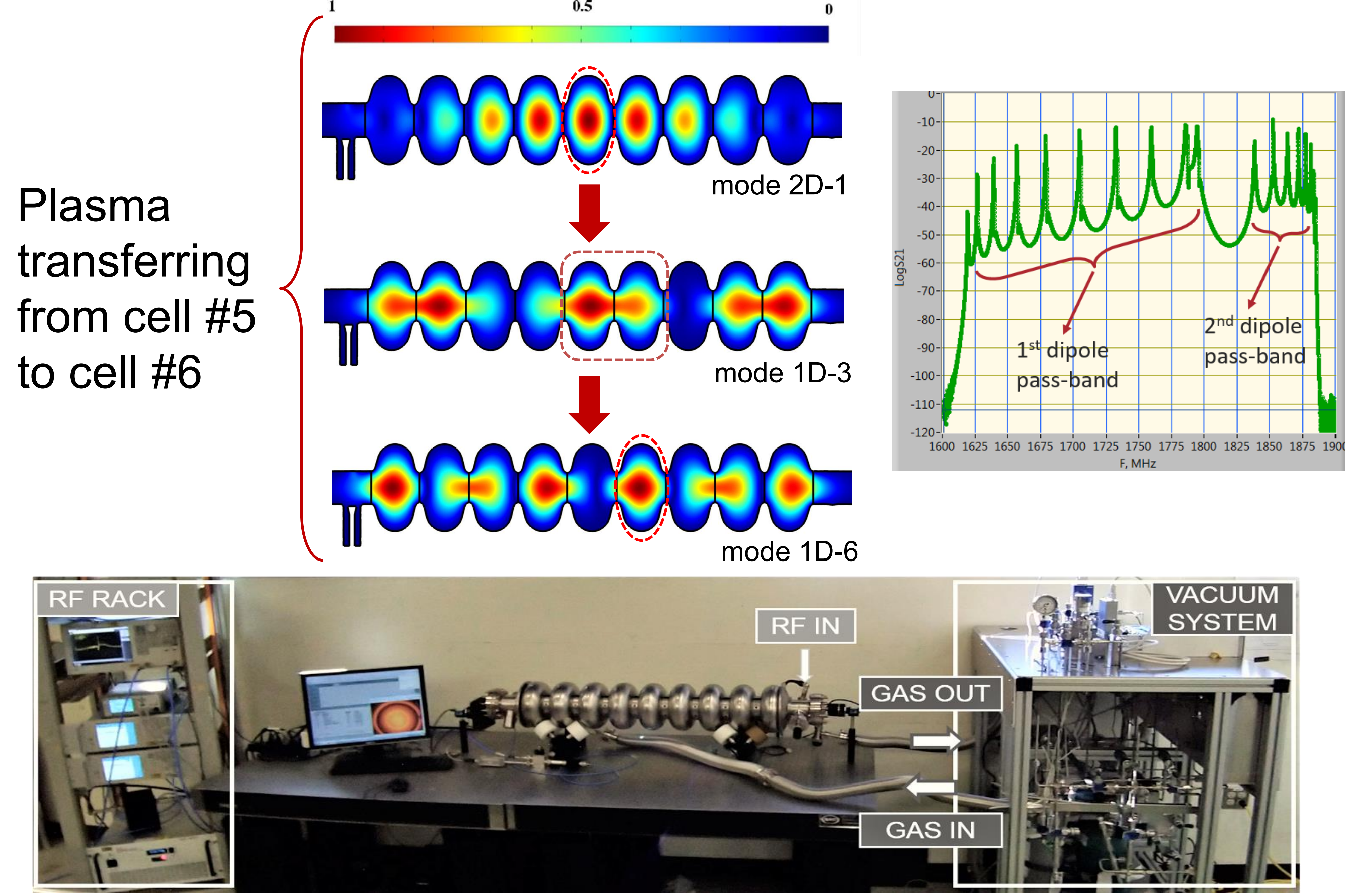

\section{Removal Studies}

Cavity iris: contaminated with permanent marker (ink: C compound)
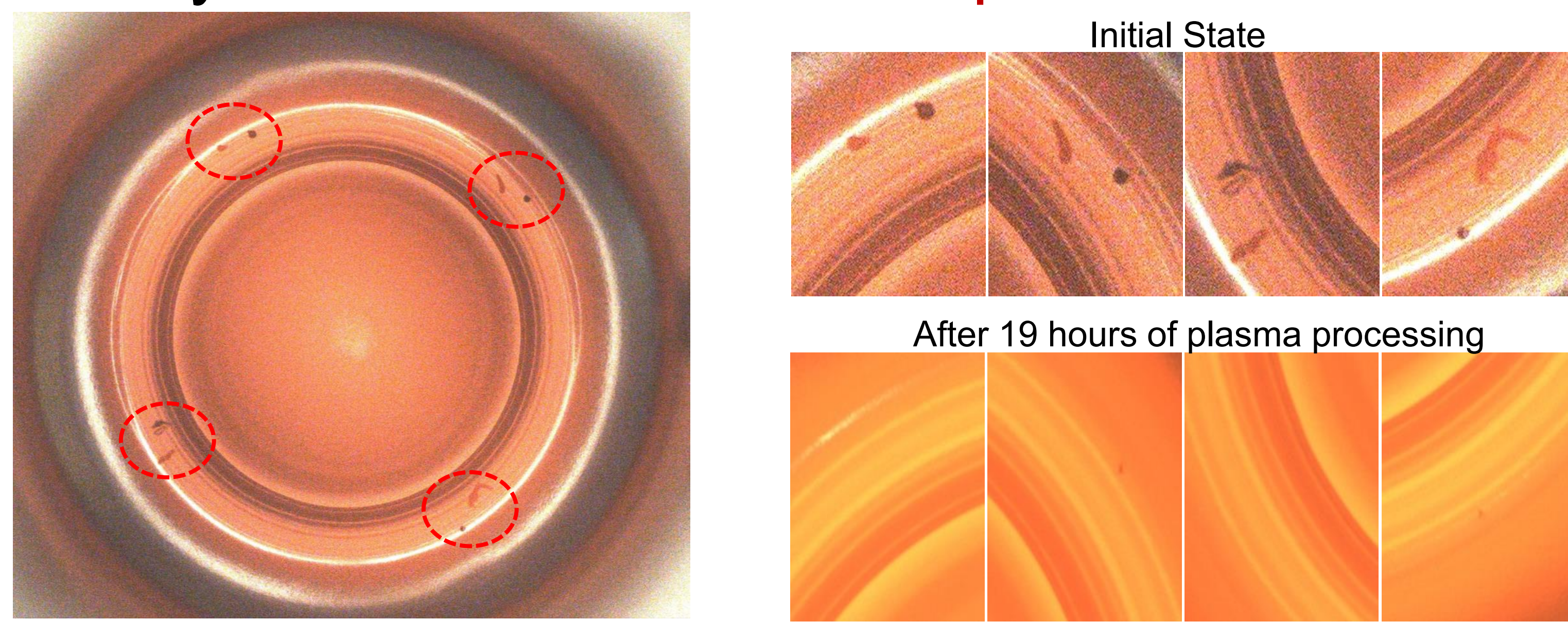

Developed a first recipe in terms of pressure, $\mathrm{O}_{2}$ percentage, plasma density.

\section{Effect of plasma on Q- factor of $\mathrm{N}$-doping}

RF test proved that plasma preserves the high $Q$ and quench field of $\mathrm{N}$-doped cavity

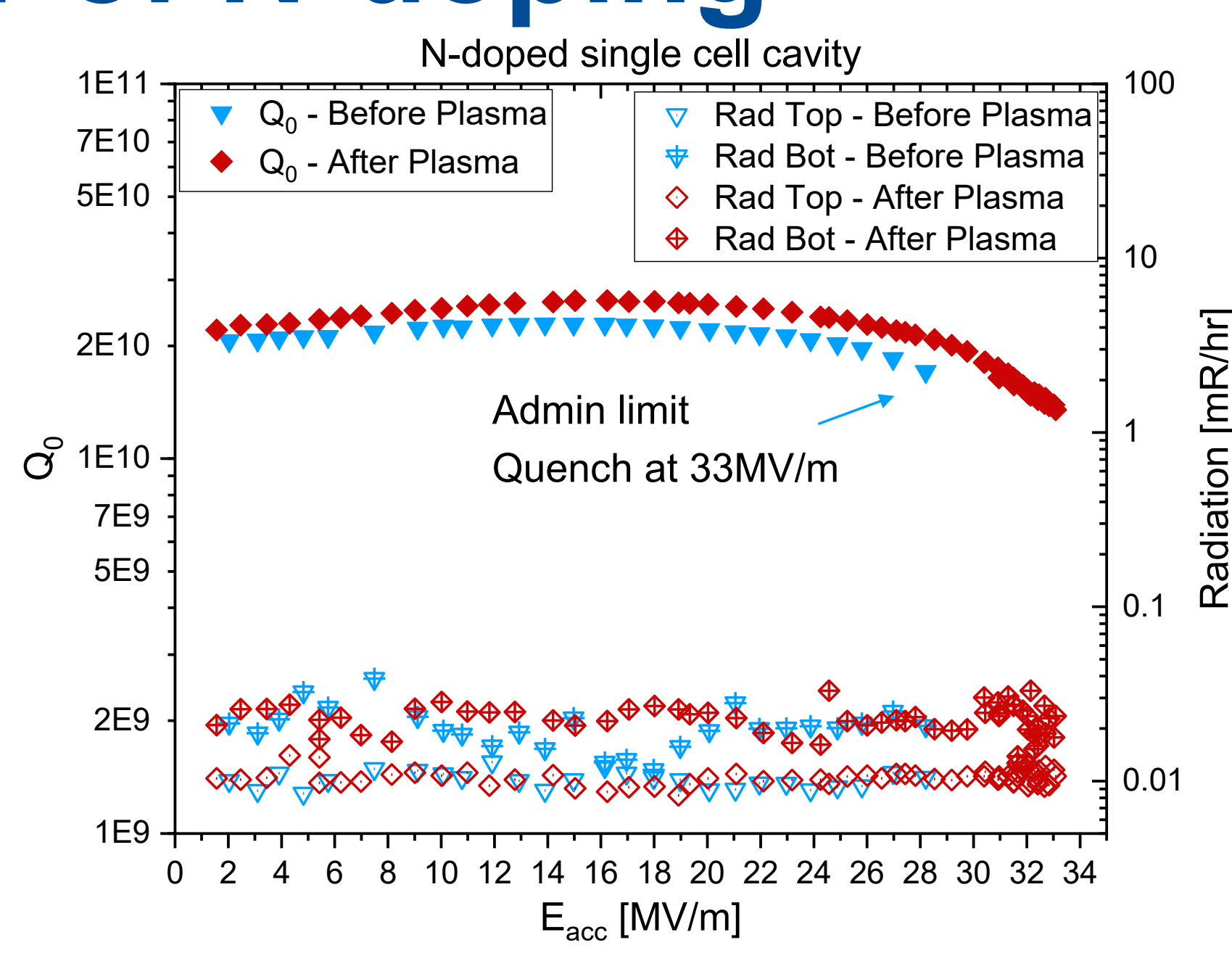

Studies on Contaminated Cavities

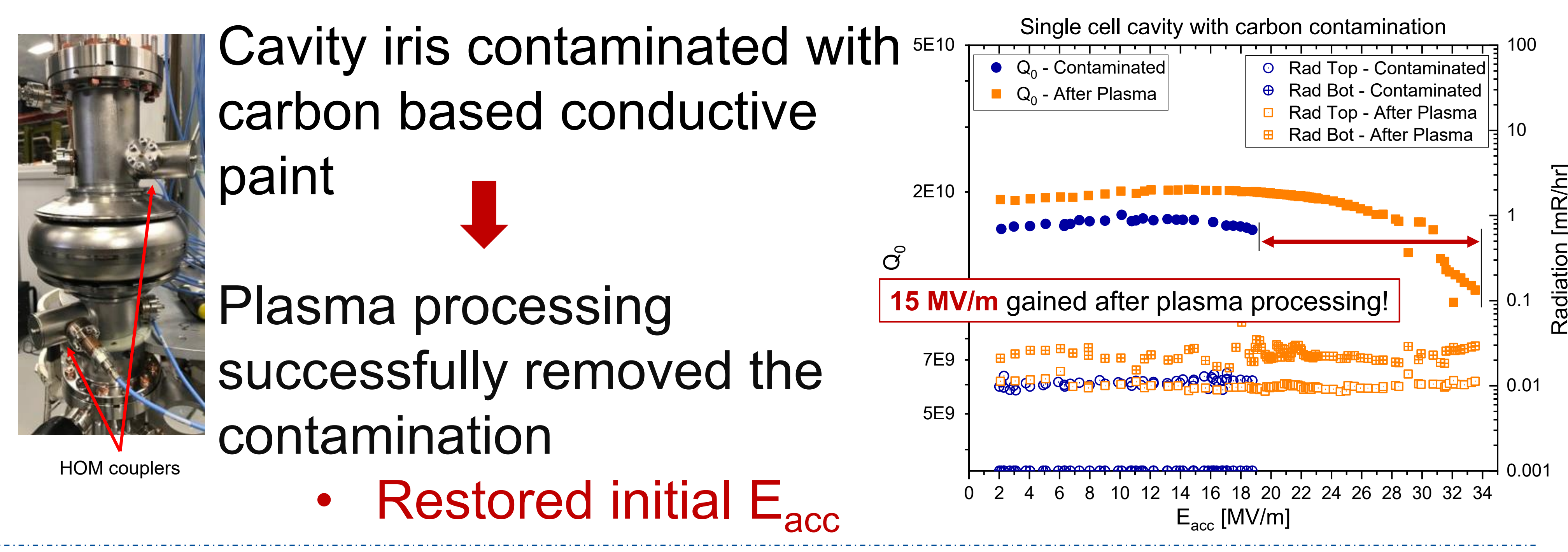

Two 9-cell cavities with natural FE:

- $1^{\text {st }}$ cavity: no increase in performance, FE may be not Crelated

- $2^{\text {nd }}$ cavity: complete removal of FE after plasma processing

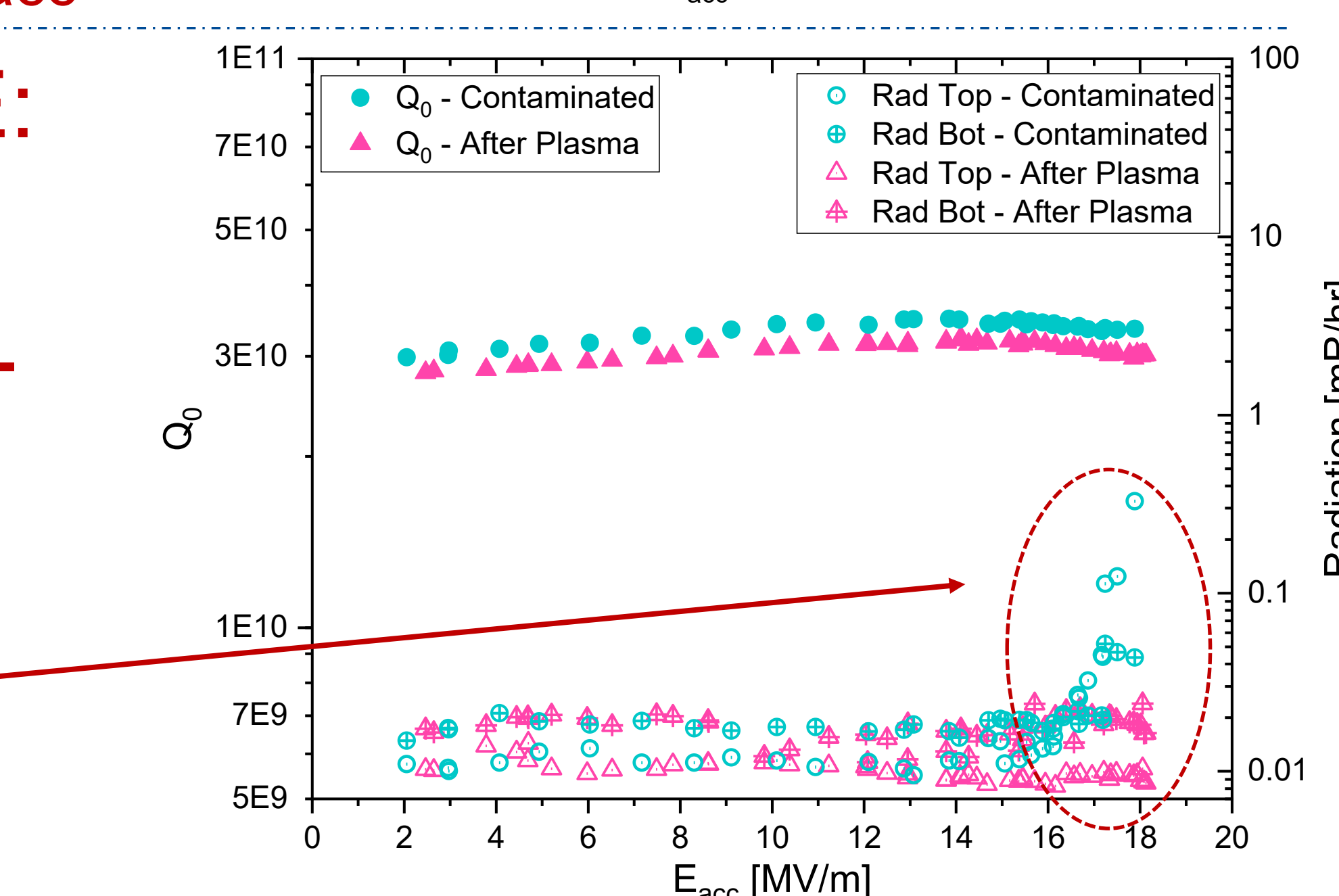

Simulated Vacuum failures

- Vented inside clean room: plasma processing completely removed FE

- Vented outside clean room: no or moderate increase in the performance

$$
\begin{aligned}
& \text { Collect particles introduced during the } \\
& \text { venting to understand contaminant } \\
& \text { composition }
\end{aligned}
$$

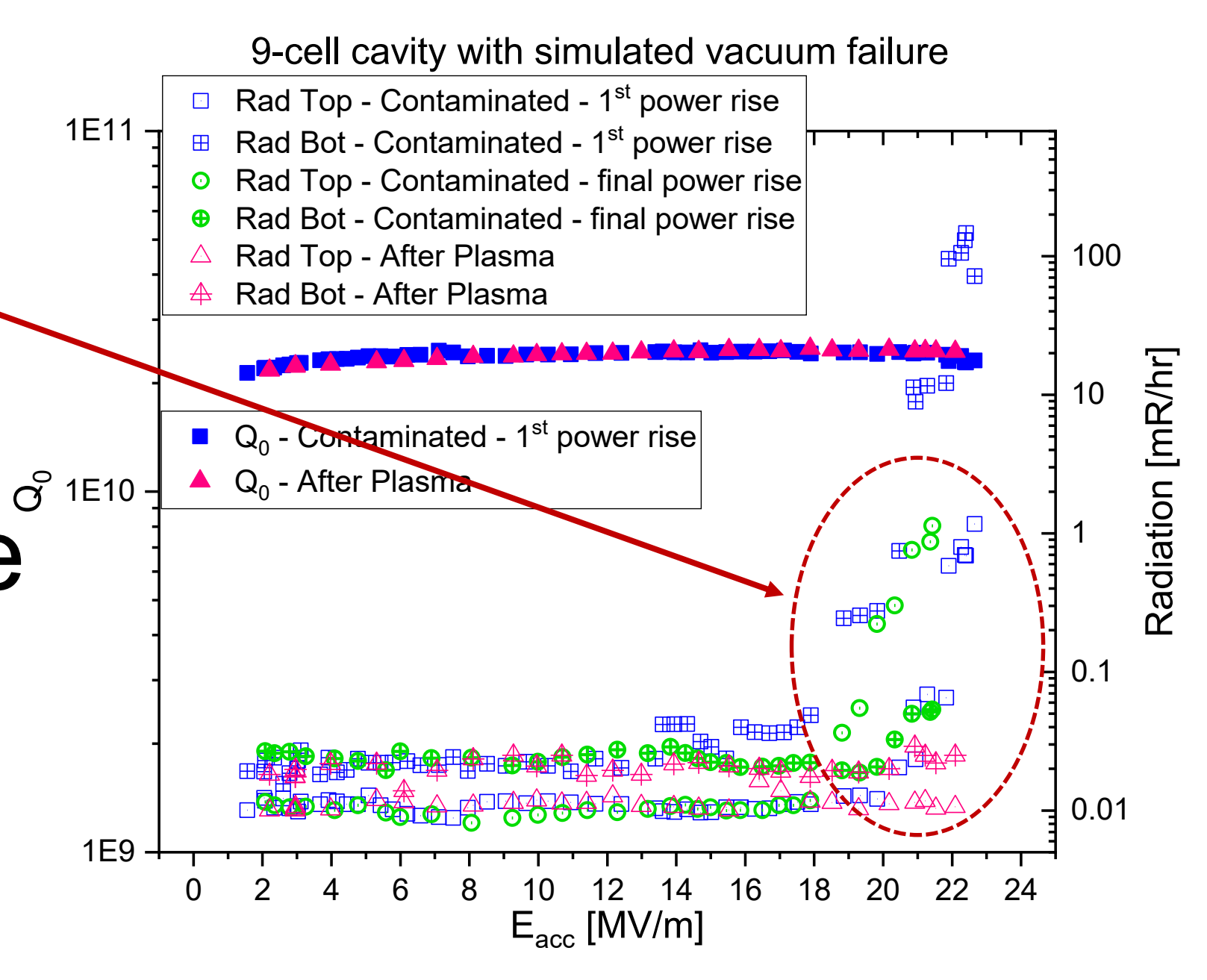

\section{Conclusions}

- HOMs method: substantially reduces the forward power necessary to ignite plasma discharge

- Successfully removed $C_{x} H_{y}$ contamination and identified a first working recipe

- RF tests before and after plasma cleaning show:

- Plasma processing preserves high $Q$ and quench field of $\mathrm{N}$-doped cavity

- Plasma processing can remove field emission and increase quench field in cavities with various carbon-based contaminations

Current and future work:

- Optimize plasma parameters (pressure, duration, $\mathrm{O}_{2}$ percentage, plasma density) to increase efficiency

- Acquire more statistics on the effectiveness of plasma processing applied to natural field emission

This manuscript has been authored by Fermi Research Alliance, LLC under Contract No. DE-AC02-07CH11359 with the U.S. Department of Energy, Office of Science, Office of High Energy Physics 